Research article

\title{
Child-Adult Relationship Enhancement (CARE): An evidence-informed program for children with a history of trauma and other behavioral challenges
}

\author{
Robin H. Gurwitch ${ }^{\mathrm{a}, *}$, Erica Pearl Messer ${ }^{\mathrm{b}}$, Joshua Masse ${ }^{\mathrm{c}}$, Erna Olafson ${ }^{\mathrm{d}}$, \\ Barbara W. Boat ${ }^{\mathrm{e}}$, Frank W. Putnam ${ }^{\mathrm{f}}$ \\ a Duke University Medical Center, Center for Child and Family Health, 1121 West Chapel Hill Street, Suite 100, Durham, NC 27701, USA \\ b Cincinnati Children's Hospital Medical Center, 3333 Burnet Ave, MLC 3008, Cincinnati, OH 45229, USA \\ c University of Massachusetts Dartmouth, 285 Old Westport Road, LARTS 390, North Dartmouth, MA 02747-2300, USA \\ d Cincinnati Children's Hospital Medical Center, University of Cincinnati Medical School, 311 Albert Sabin Way, Floor R, Cincinnati, OH \\ 45229, USA \\ e University of Cincinnati Academic Health Center, Cincinnati Children's Hospital Medical Center, 311 Albert Sabin Way, Floor R, \\ Cincinnati, OH 45229, USA \\ ${ }^{\mathrm{f}}$ University of North Carolina at Chapel Hill, 422 Brushy Fork Lane, Stuart, VA 24171, USA
}

\section{A R T I C L E I N F O}

\section{Article history:}

Received 6 March 2015

Received in revised form 12 October 2015

Accepted 19 October 2015

Available online 22 November 2015

\section{Keywords:}

Evidence-informed

Parent-Child Interaction Therapy (PCIT)

Child maltreatment

Child behavior problems

Implementation

Dissemination

\begin{abstract}
A B S T R A C T
Child maltreatment impacts approximately two million children each year, with physical abuse and neglect the most common form of maltreatment. These children are at risk for mental and physical health concerns and the ability to form positive social relationships is also adversely affected. Child Adult Relationship Enhancement (CARE) is a set of skills designed to improve interactions of any adult and child or adolescent. Based on parent training programs, including the strong evidence-based treatment, Parent-Child Interaction Therapy (PCIT), CARE was initially developed to fill an important gap in mental health services for children of any age who are considered at-risk for maltreatment or other problems. CARE subsequently has been extended for use by adults who interact with children and youth outside of existing mental health therapeutic services as well as to compliment other services the child or adolescent may be receiving. Developed through discussions with Parent-Child Interaction Therapy (PCIT) therapists and requests for a training similar to PCIT for the non-mental health professional, CARE is not therapy, but is comprised of a set of skills that can support other services provided to families. Since 2006, over 2000 caregivers, mental health, child welfare, educators, and other professionals have received CARE training with a focus on children who are exposed to trauma and maltreatment. This article presents implementation successes and challenges of a trauma-informed training designed to help adults connect and enhance their relationships with children considered at-risk.
\end{abstract}

๑) 2015 Elsevier Ltd. All rights reserved.

\footnotetext{
is The dissemination of CARE in Delaware was funded, in part, by the following sources: Delaware Child Care Development Fund, Race to the Top-Early Learning Challenge grant, and SAMHSA Children's Mental health Initiative/System of Care. The funding sources had no involvement in the preparation and submission of this manuscript.

* Corresponding author.
} 


\section{Introduction}

In 2012, the U.S. Department of Health and Human Services, Administration for Children and Families, reported that over two million children were documented as founded reports of child maltreatment; approximately 680,000 were unique victims. Physical abuse and neglect is the most common type of abuse (U.S. Department of Health and Human Services, 2013). Annually, reports indicated approximately 400,000 children are in the foster care system (Child Welfare Information Gateway, 2013), which presents additional challenges. When children experience maltreatment, psychosocial development is negatively impacted, especially regulation of emotions, control of impulses and behaviors, and the ability to have healthy and happy relationships (Putnam, 2006). Each year, several million children are referred for services to address these consequences of child maltreatment (U.S. Department of Health and Human Services, 2013). The referrals for services are generally for one of two reasons: (1) prevention of future child maltreatment referrals or (2) improvement in the conditions that brought the child to the attention of the child welfare system.

In recent years there has been a rapid proliferation of both evidence-based and promising treatments for children with maltreatment and trauma exposures. The Kaufman Best Practices Project (2004) identified three practices which meet standards for a strong evidence-based intervention: Trauma-Focused Cognitive Behavioral Therapy for Child Sexual Abuse (now extended to include all forms of abuse), Abuse-Focused Cognitive Behavioral Therapy for Physical Abuse (renamed: Alternatives for Families: A Cognitive Behavioral Therapy) and Parent-Child Interaction Therapy (PCIT). As Chaffin and colleagues note (Chaffin et al., 2004), parent (caregiver) involvement and parent training are included in most all referred services. The three best practices all include parents/caregivers.

Trauma-Focused Cognitive Behavioral Therapy (TF-CBT), with multiple randomized trials documenting effectiveness for children aged 3-18 and their caregivers (Cohen, Mannarino, \& Deblinger, 2006, 2012; de Arellano et al., 2014), includes both parallel and joint sessions. Parental participation is seen as central to recovery. Parenting skills are addressed early in treatment and throughout, and psycho-education for both parent and child address posttraumatic symptoms and behaviors. Other standard trauma components for both parent and child include cognitive processing, affect regulation, exposure therapy, sharing of the trauma narrative, and safety planning. Research shows that TFCBT reduces posttraumatic stress and improves caregiver support for their traumatized children (de Arellano et al., 2014).

AF-CBT, Alternatives for Families-A Cognitive Behavioral Therapy (Kolko \& Swenson, 2002), targets children aged 5-17 and their caregivers/parents with histories of child physical abuse and concurrent emotional abuse such as verbal aggression. Children and parents are seen both individually and together, and components for each include psycho-education about the impact of abuse and conflict, cognitive processing, and affect regulation training. Children are taught social and interpersonal skills and supported through exposure work about traumatic events, and parents are trained in non-violent child behavior management strategies. In joint sessions, caregivers clarify their responsibility for the abuse and families develop safety plans. Families practice improved communication skills and non-coercive family problem-solving skills in sessions and with home practice assignments. Research comparing AF-CBT with routine community services showed lower rates of parent child physical abuse recidivism and improvements in child externalizing behaviors (Kolko \& Swenson, 2002).

Parent-Child Interaction Therapy (PCIT) is an evidence-based treatment developed to decrease externalizing behavior problems in young children (2-7 years of age) and to improve the parent-child relationship (Brinkmeyer \& Eyberg, 2003). An assessment-driven intervention, PCIT is unique in parent management training and therapy programs as it involves direct coaching of the parent and child. Studies have consistently found improvements in child behaviors reported by caregivers on standardized measures (Chase \& Eyberg, 2008), reductions in parenting stress (Harwood \& Eyberg, 2006) and depression (Ho, 2004), and generalization to school settings (Funderburk et al., 1998) as well as to improvements in behaviors of untreated siblings (Brestan, Eyberg, Boggs, \& Algina, 1997). Recent data suggests PCIT can be effective in reducing some forms of childhood anxiety (e.g., separation anxiety disorder; Choate, Pincus, Eyberg, \& Barlow, 2005). Although not specifically developed as a treatment for children with a child abuse and neglect history, a randomized controlled trial of PCIT with children ages 4-12 years and their parents/caregivers with history of physical abuse/neglect, examined recidivism rates of child physical abuse at two years post-treatment. For the PCIT-only condition, the study found recidivism rates at less than twenty percent, which is significantly less than the norm (Chaffin et al., 2004). Furthermore, Pearl and her colleagues have found a reduction in trauma symptoms in children who received PCIT (Pearl et al., 2012). These findings have increased the demand for PCIT in agencies, which serve children with a history of child abuse and neglect.

Although these three programs are being widely disseminated across the United States, many children who are at risk for maltreatment or at risk for mental health problems do not present or qualify for mental health services. Behavior problems also place children at risk for stress in any child-adult relationships, including problems which result in failed foster care placements (Rubin, O’Reilly, Luan, \& Localio, 2007). A void existed for services to enhance the child-adult relationships for these children, and potentially reduce risk for maltreatment or other behavioral and relationship concerns; CARE (Child-Adult Relationship Enhancement) was the created to fill this void.

\section{Parent Training Programs, PCIT, and CARE}

Evidence-based parent training programs such as Helping the Noncompliant Child (Forehand \& McMahon, 1981), Incredible Years (Webster-Stratton \& Reid, 2003), Parent Management Training-Oregon Model (Forgatch, Bullock, \& Patterson, 2004) and Parent-Child Interaction Therapy (Eyberg \& Robinson, 1982) have many attributes in common. Each works to 
change the parent-child relationship by replacing overly permissive and/or harsh inconsistent discipline with more structured, positive behavior management skills and strategies (Pearl, 2009). Building on Hanf's two-stage program, parents learn and practice skills to improve family interactions (Eyberg \& Matarazzo, 1980). These parenting programs, especially PCIT form the basis for the CARE (Child Adult Relationship Enhancement) program; therefore, an overview of the components of PCIT is important to understanding the development and components of CARE. PCIT involves two phases (Child-Directed Interaction, CDI and Parent-Directed Interaction, PDI), each of which includes a teaching session and several coaching sessions. During the teach sessions, parents are taught specific skills which they must master prior to moving forward in treatment. Skills taught are summarized as PRIDE skills with each letter identifying a particular skill designed to address goals of CDI. These include Labeled Praise (P), Reflect (R), Imitate (I), Describe (D), and Enjoy (E) with training in selective attention for specified inappropriate behaviors. They are also taught to avoid giving commands, asking questions, and using critical language as these can undermine a positive relationship. The in vivo coaching sessions are designed to help meet skill mastery goals. PCIT is an extremely transparent treatment, with feedback about progress shared with the parents at each session. The goals of this first phase of PCIT are consistent with improving the parent-child positive relationship. They include: increasing positive child behaviors, improving frustration tolerance and anger management, improving social skills, building self-esteem, improving organization and attention skills, and reducing child negative attention-seeking behaviors.

The second phase of PCIT, the Parent-Directed Interaction (PDI) focuses on increasing consistency, predictability and parents' ability to follow-through with an effective positive discipline program. This phase of treatment is designed to improve parents' abilities to give effective commands, set appropriate limits, implement contingency management strategies, problem-solve discipline situations and decrease remaining negative child behaviors.

\section{The CARE Program}

Child-Adult Relationship Enhancement (CARE) has a unique history among caregiver-focused interventions. In 2006, the concept for CARE developed from a series of collaborative discussions between Parent-Child Interaction Therapy (PCIT) trainers and PCIT therapist trainees. The PCIT therapist trainees were participating in a research study conducted by the Cincinnati Children's Hospital Medical Center on the feasibility and sustainability of implementing PCIT within their respective agencies. The purpose of the project was to develop expertise in transferring evidence-based and evidenced-informed treatments for children and adolescents with trauma histories to community mental health providers who often serve special or culturally diverse clients.

As part of PCIT training, therapists were involved in a year-long consultation process. During PCIT consultation sessions with "Medical Center" trainers, PCIT therapist trainees frequently reported the desire to share specific PCIT skills with others who interacted with their clients. They recognized that PCIT was a therapeutic intervention, but stressed a belief that the skills could be helpful, even therapeutic, to others interacting with their clients. The consistent message from all of the therapist trainees was that basic skills that enhance positive interactions between children and adults "should be in the water" and available to everyone. For example, using PCIT skills, one therapist coached a bus driver to give a Labeled Praise to her client when she stayed in her seat. Another, using PCIT principles of how to give effective commands, coached a receptionist to say to the child in PCIT treatment, "Please close the door gently" rather than "Quit slamming the door." With these and many similar examples, the "Medical Center" training team embarked on a project to incorporate their successful adaptations of the core components of PCIT and ideas from other parent management training programs, and developed the CARE model to be used by any adult interacting with children and adolescents in any setting.

Whereas PCIT is an evidenced-based therapy for children 2-7 years of age, CARE is not a therapy program. CARE skills can be implemented by any adult interacting with a child between the ages of 2 and 18 years of age. CARE training does not address extreme disruptive behavior or aggression, which may require a therapeutic intervention. CARE training was designed to be used more as a prevention model for children at risk for maltreatment or other behavioral concerns, or to complement ongoing therapy services. PCIT incorporates social learning principles to address three areas important in the lives of children with history of child maltreatment: (a) disrupting coercive cycle, (b) improving parent-child relationships, (c) effective alternatives to physical discipline (Urquiza \& McNeil, 1996). CARE skills also support these goals and may reduce risk for maltreatment and other behavioral concerns. If adults learn ways to quickly and effectively engage in a positive relationship with the child or adolescent, CARE developers theorized that the children and adolescents will be more likely to interact with the adult in an appropriate manner, including improving compliance. The CARE training also contains a trauma education component to contextualize the use of CARE skills with the kinds of behaviors and problems exhibited by many children exposed to trauma and maltreatment.

Over time, the CARE manual has been revised in response to input from new "Medical Center" PCIT team members and feedback from trainees in a variety of settings. Thus, CARE reflects a collaborative enterprise with trainers and trainees from a range of agencies including residential treatment centers, foster care agencies, transitional living programs, child care settings, medical care settings, and educational settings.

\section{Care Training}

The Curriculum Guide for CARE Trainers was developed in 2006 and updated as recently as 2015; it contains step-by-step guidelines on how to conduct a CARE training. There are training guidelines and handouts for use with both children and 
adolescents. CARE trainers are required to first complete PCIT, PMT-O, IY, or Helping the Noncompliant Child training so that they have a clear knowledge of the guiding principles and skills upon which the CARE training were based. The CARE training starts with an introduction of how CARE was developed and the goals and objectives of the training. The impact of trauma, particularly child maltreatment, on child and adolescent behaviors and risks for problems in child-adult relationships is discussed. Examples from this discussion continue to be used throughout CARE training. The CARE participants watch a video presentation of a CARE trainer playing the role of a foster parent and interacting with a non-clinical 4-yr-old child in two situations: (1) typical foster parent engaging with a young child, and (2) foster parent using CARE skills engaging with the same child. The participants are asked about observations of the connection in each video clip. Typical observations include that the child speaks much more, seems more engaged and appears to be enjoying the interaction more when the trainer is following the child's lead, avoiding questions and directions and praising the child. Participants are then taught the first components of CARE (the "P's and Q's", and strategic ignoring) when following a child's lead to connect and engage with them. The "Q" skills are similar to those in PCIT as is strategic ignoring. These include: Quash the need to lead, Quit unnecessary questions, and Quiet the criticisms. The "do" skills in CARE have simplified from those in PCIT to include what is termed "the 3 P's": Praise (Labeled praises for appropriate behaviors), Paraphrase (use of reflective listening techniques), and Point Out (attending to positive actions in the child or adolescent). Didactic materials are interwoven with experiential exercises to improve learning. For example, after information is provided for each of the "do" skills and for "strategic ignoring," participants are instructed to practice the skills with a partner at their seats, using toys and handouts to assist them. During this time, trainers briefly observe and provide feedback to each dyad. Trainers teach participants how to monitor the "do skills" using tally marks on record sheet listing the P's and Q's which was modified from the more complex PCIT coding forms. Participants break into small groups of approximately 4-6 people and practice all of the "P skills" together, roleplaying as adults and children with toys. Remaining group members practice recording each dyad. The dyads rotate so that each group member has a chance to monitor the skills and play the role of an adult and child. A trainer provides live feedback to each person and group members work to give at least 5 of each of the P skills with less than 3 of the Q's in a 3 min period of time. Trainers briefly coach participants who may be struggling with a particular skill by giving ideas and reinforcing implementation of these suggestions. Following this practice and discussion of how to use these skills with children, CARE training shifts to a focus on how to improve relationships when compliance to task is the goal. Participants are taught the importance of giving good commands or directions, similar to what is taught in PCIT and other parent management training programs. As with the P's and Q skills, participants then put didactic learning into active practice. Role-play activities are utilized so that participants can practice the skills and receive feedback from trainers. In sum, CARE training is a blend of short didactic presentations with much "hands-on" practice and live feedback and discussion.

CARE training sessions generally are between 3 and $6 \mathrm{~h}$, depending on the number of participants as well as agency goals for the training. Many agencies who have received CARE instruction chose to do CARE training for 3-4 $\mathrm{h}$ on one date with a follow-up 2-3 h training at a later date to allow participants to implement CARE skills in their settings and discuss their experiences. This format is ideal for (a) facilitating discussion at the later training regarding success and challenge stories and (b) maintaining skills.

\section{CARE and Trauma Interventions}

Although involvement of parents or caregivers serving in a parenting role in evidence-based trauma treatments for children is growing, few provide ideas and skills for other caregivers or adults that may interact with children and adolescents with a history of maltreatment (e.g., teachers, residential treatment staff, other clinic staff, etc.). Thus trauma-responsive milieu trainings such as Sanctuary (Bloom, 2005; Rivard et al., 2003) or Think Trauma (NCTSN.org/Think Trauma, 2015) have been developed. These models are widely trained in residential and juvenile justice programs, with anecdotal accounts reporting widespread success in transforming agency cultures. However, no randomized studies exist yet documenting their effectiveness. The same is true for trauma-focused training program developed and disseminated through The National Child Traumatic Stress Network (2010) for use specifically with foster parents, Caring for Children Who Have Experienced Trauma: A Workshop for Resource Parents. The CARE program seems to add to this growing need for helping adults interacting with a child or adolescent to increase their ability to help the children or adolescents reach their potential.

\section{CARE Implementation and Dissemination Efforts}

\section{Training Activities}

Since CARE was first unveiled almost a decade ago, there have been numerous requests for training. Over 2,000 individuals have been trained in CARE across the country. Caregivers (biological, foster, and kinship, and adoptive caregivers) have been recipients of CARE training as well as being taught CARE by their therapists as a part of mental health interventions. Mental health providers are one of the largest groups who have participated in CARE training. Most have not been trained in PCIT, but see CARE as being a "value-added" to the mental health work they do with families, especially with children and adolescents with trauma histories. Allied Health professionals (e.g., Occupational and Physical Therapists, Speech and Language pathologists, Child Life Specialists) have been another audience for CARE training. Feedback from participants indicated they believe CARE will make a positive difference in the work they do with children and adolescents. For many 
years, CARE has been provided to medical and psychology students and residents at Cincinnati Children's Hospital Medical Center. Physicians, nurses, and psychologists have also been included in the trainings and then report they are implementing CARE with families. Although systematic data collection is lacking, training evaluations and qualitative reports of those implementing CARE have been positive. For example, a physician who conducts child sex abuse examinations noted that two to three minutes of CARE skills with her young patients appears to reduce their anxiety and improve the ability to perform a difficult procedure. CARE training has been provided to those working in the field of child abuse and neglect across many states. Participants have included child protective services case workers, case managers, providers, and shelter workers and shelter staff. Additionally, those working in domestic violence centers, substance abuse treatment programs, and inpatient units have participated in CARE training. School personnel (teachers and staff) and early childcare providers have regularly participated in CARE trainings provided across the United States. Following the trainings, they report the ability to quickly implement services into the classroom setting. Recently, novel populations have been involved in CARE training such as military chaplains, military prevention team members, and Family Advocacy Program personnel. These personnel have stated a belief that CARE should be included in deployment cycle programming for any military family with children (Gurwitch, Fernandez, Pearl, \& Chung, 2013).

In sum, there are a myriad number of populations that have or could participate in CARE training-it is appropriate for any adult who ever interacts with a child or adolescent.

\section{CARE Dissemination Example: Delaware CARE Initiative}

The largest systematic dissemination of CARE has been conducted in the State of Delaware. In 2008, the State of Delaware's Division of Prevention and Behavioral Health Services (DPBHS) received a SAMHSA child mental health initiative grant focused on increasing the availability of evidence-based treatments for young children (under age 6) and their families. Through this initiative, Delaware's B.E.S.T., the division sought to create, maintain and sustain evidence-based mental health interventions by developing a continuum of services for the early childhood population. Delaware chose PCIT as the primary treatment of focus. PCIT was trained statewide to mental health practitioners across an array of contexts including intensive home-based (e.g., twice/week with high-risk families), community mental health agencies, and within a state-run day treatment and inpatient setting for children (see Beveridge et al., 2015 for full review of the PCIT dissemination and implementation process in Delaware). In addition to PCIT, Teacher-Child Interaction Training (TCIT), a classroom adaptation of PCIT maintaining the core principles of the model (e.g., teach sessions, coaching, data driven), was disseminated state-wide to early childhood educators.

Given its strong PCIT foundation and a desire to reach more adults working with young children, CARE was chosen as a logical extension of the milieu of evidence-based treatments being offered to fill a void often overlooked by large training initiatives - individuals in non-clinical positions who interact with children in health settings, yet often lack the resources and training to implement theoretically-sound and practical behavior management skills. A Delaware-based trainer with extensive PCIT training and clinical experience originally learned the CARE model by attending a workshop at the "Medical Center" and remained in consultation with the training team as CARE implementation progressed in Delaware. CARE training followed a train-the-trainer model. To cover community-based CARE workshops, two licensed mental clinicians on the B.E.S.T team were trained to teach the CARE model. Once CARE training was completed and consultation in place to assure fidelity, CARE was introduced in Delaware in 2011. CARE was disseminated to individuals in a variety of settings to a variety of individuals, including community mental health staff (approximately 40 individuals) milieu staff at an acute mental health setting (approximately 40 individuals), speech and occupational therapists (approximately 20 individuals), and support specialists working with women and families at dual diagnosis crises centers (approximately 20 individuals).

To further increase community dissemination, a large-scale effort was undertaken to train early childhood educators housed in pre-K centers in CARE. The rationale for CARE in the classroom settings was based on scientific literature of the issue. Classroom behavior difficulties among young children is becoming more prevalent with research demonstrating between $16 \%$ and $30 \%$ of children displaying behavioral issues (Kupersmidt, Bryant, \& Willoughby, 2000) with a comparable number of children meeting diagnostic criteria for a formal disruptive behavior disorder (Webster-Stratton \& Hammond, 1998). Given that teachers often report they feel ill-equipped to manage behavior issues in the classroom (Tiano \& McNeil, 2006) and coupled with Delaware's rank as fourth in the nation in rate of expulsion of pre-school children from public programs because of behavior problem (Gilliam, 2005), CARE was sought after as a evidence-informed program to assist early educators in developing and improving upon behavior management skills.

CARE was disseminated in conjunction with an Early Childhood Mental Health Consultation (ECMHC) team, a group of licensed mental health professionals responsible for providing an array of services to early education sites focusing on lowincome schools with at-risk children. Specifically, ECMHC's goal is to promote the social-emotional well-being of students while looking to reduce the likelihood of expulsion for the children considered at the highest risk. Prior to learning the CARE model, each consultant received the standard PCIT didactic training (i.e., $40 \mathrm{~h}$ of training) to become familiar with the core components of the treatment model. In addition, following a one-day CARE trainer workshop, each consultant was observed conducting a CARE workshop and given specific feedback. Finally, each CARE consultant participated in six monthly consultation calls with the lead trainer. In sum, 9 CARE consultants were fully trained to deliver CARE workshops. As of January 2015, 1,288 early learning professionals have been trained in CARE through over 100 workshops incorporating adult learning principles. The trainings took place on Saturdays at various locations across the state, central to the county. 
Although pre-post workshop data is currently being examined, training satisfaction and improved competency and use of skills are very promising.

\section{Research Activities}

Although CARE is evidence-informed, there are no published manuscripts to date. However, a few studies in Cincinnati and Philadelphia are underway; CARE training is currently being studied with foster parents with goals of reducing failed placements, reducing externalizing symptoms, and decreasing parental stress. CARE has also been studied with pediatric residents (Spector et al., 2012). Outcomes evaluated included increased beliefs that it is a pediatrician's role to discuss parent-child related issues, increased comfort level in modeling behaviors taught in the CARE workshop, increased comfort in discussing parent-child issues with parents and improved ability to access resources to help parents who struggle with how to discipline their children. Additionally, CARE is being implemented at schools and child development centers serving families stationed at Tripler Army Medical Center, Hawaii. Goals of this endeavor include a reduction in negative behavior reports, expulsions, suspensions of students and endorsement of improved classroom management and environment by teachers (Gurwitch et al., 2013).

As research efforts continue, satisfaction information was obtained from participants in CARE training. Evaluations collected between 2008 and 2015 through Cincinnati Children's Hospital Medical Center surveyed over 100 trainees in various professions including child protection staff, forensic interviewers, home visitors, and residential treatment care staff. 99.2\% of the CARE participants $(n=132)$ answered "yes" to the question: Do you think this training will change the way you practice/interact with children and adolescents? 98\% of the CARE participants $(n=120)$ answered "yes" to the question: Would you recommend this training to a colleague?

\section{CARE Successes and Challenges}

Successful dissemination of CARE has been contingent on several factors. In Delaware as well as in other settings such as in Tripler Army Medical Center and Cincinnati Children's Hospital Medical Center, it was beneficial to have an on-site evidencebased expert trainer with PCIT experience positioned in a role focused on training and implementing research-supported programs in the setting or across the state. As such, CARE was more easily blended with a milieu of trainings and into a preexisting training structure. Relationships with existing systems where CARE was being implemented were also essential to uptake and success. For example, in Delaware, a large portion of the first CARE trainings with mental health agencies was initiated by pre-existing relationships with the state (e.g., state contracted agencies, state-run mental health settings). Next, the proliferation of CARE across early childhood education settings was achieved in large part by partnering with an already established early childhood consultation team. At Tripler, CARE enhanced service delivery to school settings which had existing connections and trust in mental health resources which served military families. In Philadelphia, a partnership with the foster care system and child welfare agency allowed for greater uptake by foster parents as CARE was incorporated into training and available resources, giving it a "stamp of approval." Finally, in many cases, by linking CARE with entities responsible for awarding continuing education credits for attendees, professional incentive was increased, and advertising for the workshop gained greater exposure.

As a training workshop, the brevity of the CARE experience has been an implementation challenge as follow-up data was not attained subsequent to the training. A lack of follow-up data disallows for assessment of implementation of skills in any field setting. One solution that may alleviate this issue is to train an on-site trainer to train, monitor, coach, and collect data on the implementation at a respective agency. The agency trainer would be required to attend a training-the-trainer workshop, have a strong behavioral background and experience with evidence-based techniques, and stay in consistent consultation with the expert trainer while ensuring that the CARE skills are incorporated at the site. This approach would also allow for implementation science variables to be incorporated and assessed in a continuing way to assess for uptake, fidelity, sustainability in addition to desired outcome variables. Another potential solution is to conduct half-day booster workshops in agency settings which would include in vivo coaching and feedback to participants who are using CARE skills. Alternatively, a subsequent site visit following the training focused on skill applications would increase ecological validity. Although these solutions would assist with the workshop-to-real life transfer of skills, it would also require additional trainer time and as well as needing to have coverage during the weekday workshop for involved staff.

Another challenge inherent in the CARE model is buy-in and sustained uptake of the skills. While evaluations at the completion of CARE workshops are overwhelmingly positive, there is currently no systematic data which provides information about agency change after CARE is learned or if skills are actually used by those attending (either within an agency or setting or on the individual level). In speculating which agencies are more successful in sustaining CARE over time, two variables appear critical: agency support and commitment to cultural change. In Delaware, the sites that were committed to fundamentally changing the manner in which staff communicated with children coupled with strong encouragement by administration and colleagues appeared to realize the most success with the learned skills. As a proxy to this outcome, a factor that successful and committed agencies shared was attendance of both staff and administration in the workshop.

As discussed, forging partnerships with local and state organizations was critical to the success of CARE within any setting; however, lack of visible partnerships or support can also be a challenge to dissemination. For example, despite a strong effort and the forging of a relationship with an agency involved with training foster parents in Delaware, there was 
a lack of participation in CARE with the supposition being that this group is taxed with numerous responsibilities and other trainings, so the initial incentive was not present, and traction, unfortunately, lost. On the individual level, if there are not resources available to allow for boosters or to have implementation questions answered, then sustainability of any learned skills is likely to wean and eventually will disappear.

\section{Next Steps for CARE}

While the Kauffman report identified PCIT as one of three best practices in the field of child abuse treatment (2004), it also noted that training and dissemination efforts have been limited and described a disturbing chasm between best practice and everyday practice in community settings. Recommendations subsequently included the exploration of new approaches to promote dissemination and implementation of evidence based practices in the treatment of child abuse and other mental health concerns (Fixsen, Naoom, Blasé, Friedman, \& Wallace, 2005). This recommendation could also extend to evidence-informed programs such as CARE to address child maltreatment as well as those children at risk for abuse. The Institute for Healthcare Improvement (IHI) (2003) offered a promising model of training and implementation, referred to as the Breakthrough Series Collaborative (BSC). This innovative approach focuses on collaboration between sites interested in adopting new practices and addresses organizational barriers to implementation and sustainability. The National Center for Child Traumatic Stress (NCCTS) has pioneered the adaptation of the BSC methodology to best practices in mental health treatment, conducting dozens of Learning Collaboratives (LC) nationally on varied treatments for child traumatic stress (Ebert, Amaya-Jackson, Markiewicz, \& Burroughs, 2008). Currently, CARE is beginning to be woven into the PCIT LCs in North Carolina. The collected feedback and data on this new addition will be important in moving CARE from an evidenceinformed practice to an evidence-based set of skills. Comparisons of outcome data with and without the CARE component will be important to determining the utility of CARE in training activities.

Overall, CARE has served as a natural adjunct to the continuum of evidence-based services offered across a variety of settings. Although CARE serves in a complementary role for mental health professionals trained in therapeutic techniques (e.g., TF-CBT, PCIT, AF-CBT), it has filled a critical gap in training non-clinical professionals as well as parents/caregivers to more effectively communicate with children and adolescents to manage behavior within a family or within the context of their occupation. In some cases, children have already experienced a continuum of evidence-based services both in the classroom (e.g., TCIT with a lead teacher, CARE by teacher and classroom aides) and in outpatient settings (PCIT), thus enhancing generalization of skills across contexts. In sum, based on anecdotal and training evaluations, CARE appears to have a direct impact on professionals and the children they interact with across a variety of settings impacted by trauma and maltreatment. Research studies scientifically evaluating outcomes of CARE for this population will be the next step in moving from an evidence-informed model to an evidence-based model. As noted, many children and families are considered at risk for maltreatment, yet do not receive services. Research examining CARE's impact as a preventative tool would allow its inclusion for recommended services for high-risk, low-service families. If data supports the principles of CARE across settings, CARE has the potential be an important addition to the tools in the toolbox for working with children with a history of trauma and maltreatment.

\section{References}

Beveridge, R. M., Fowles, T. R., Masse, J. J., Parrish, B. P., Smith, M. S., Circo, G., \& Widdoes, N. S. (2015). The dissemination and implementation of Parent-Child Interaction Therapy (PCIT): Lessons learned from a state-wide system of care. Children and Youth Services Review, 48, 38-48.

Bloom, S. L. (2005). The Sanctuary Model of organizational change for children's residential treatment. Therapeutic Communities, 26(1), 61-78.

Brestan, E. V., Eyberg, S. M., Boggs, S. R., \& Algina, J. (1997). Parent-child interaction therapy: Parent perceptions of untreated siblings. Child E’ Family Behavior Therapy, 19, 13-28.

Brinkmeyer, M., \& Eyberg, S. M. (2003). Parent-child interaction therapy for oppositional children. In A. E. Kazdin, \& J. R. Weisz (Eds.), Evidence-based psychotherapies for children and adolescents (pp. 204-223). New York: Guilford

Chaffin, M., Silovsky, J., Funderburk, B., Valle, L. A., Brestan, E. V., Balachova, T., Jackson, S., Lensgraf, J., \& Bonner, B. L. (2004). Parent-child interaction therapy with physically abusive parents: Efficacy for reducing future abuse reports. Journal of Consulting and Clinical Psychology, 72, 500-510.

Chase, R., \& Eyberg, S. (2008). Clinical presentation and treatment outcome for children with comorbid externalizing and internalizing symptoms. Journal of Anxiety Disorders, 22(2), 273-282.

Child Welfare Information Gateway. (2013). Foster Care Statistics, 2012.. https://www.childwelfare.gov/pubs/factsheets/foster.cfm. Accessed 22.02.15

Choate, M. L., Pincus, D. B., Eyberg, S. M., \& Barlow, D. H. (2005). Parent-Child Interaction Therapy for treatment of separation anxiety disorder in young children: A pilot study. Cognitive and Behavioral Practice, 12, 126-135.

Cohen, J. A., Mannarino, A. P., \& Deblinger, E. (2006). Treating trauma and traumatic grief in children and adolescents. New York/London: Guilford.

Cohen, J. A., Mannarino, A. P., \& Deblinger, E. (2012). Trauma-focused CBT for children and adolescents: treatment applications. New York/London: Guilford.

de Arellano, M. A. R., Lyman, D. R., Jobe-Shields, L., George, P., Doughterty, R. H., Daniels, A. S., Ghose, S. S., Huang, L., \& Delphin-Rittmon, M. E. (2014). Trauma-focused cognitive-behavioral therapy for children and adolescents: assessing the evidence. Psychiatry Online, 1/14-14/14.

Ebert, L., Amaya-Jackson, L., Markiewicz, J., \& Burroughs, J. (2008). The NCCTS learning collaborative model for the adoption \& implementation of evidence-based mental health treatment: NCCTS guidelines for conduction a learning collaborative. Los Angeles, CA/Durham, NC: National Center for Child Traumatic Stress and Duke University Evidence-Based Practice Implementation Center.

Eyberg, S. M., \& Matarazzo, R. G. (1980). Training parents as therapists: A comparison between individual parent-child interaction training and parent group didactic training. Journal of Clinical Psychology, 36, 492-499.

Eyberg, S. M., \& Robinson, E. A. (1982). Parent-child interaction training: Effects on family functioning. Journal of Clinical Child Psychology, 11, 130-137.

Forehand, R. T., \& McMahon, R. J. (1981). Helping the noncompliant child. New York: The Guilford Press.

Forgatch, M. S., Bullock, B. M., \& Patterson, G. R. (2004). From theory to practice: Increasing effective parenting through role-play. The Oregon Model of Parent Management Training (PMTO). In H. Steiner (Ed.), Handbook of mental health interventions in children and adolescents: An integrated developmental approach (pp. 782-814). San Francisco: Jossey-Bass. 
Fixsen, D. L., Naoom, S. F., Blasé, K. A., Friedman, R. M., \& Wallace, F. (2005). Implementation research: A synthesis of the literature. Tampa, FL: University of South Florida, Louis de la Parte Florida Mental Health Institute, The National Implementation Research Network (FMHI Publication \#231). http://nirn.fmhi.usf.edu/resources/publications/Monograph/pdf/monograph_full.pdf. Accessed 15.01.09

Funderburk, B. W., Eyberg, S. M., Newcomb, K., McNeil, C., Hembree-Kigin, T., \& Capage, L. (1998). Parent-child interaction therapy with behavior problem children: Maintenance of treatment effects in the school setting. Child E' Family Behavior Therapy, 20, 17-38.

Gilliam, W. S. (2005). Prekindergartners left behind: Expulsion rates in state kindergarten systems. Yale University Child Study Center. http://info.med.yale.edu/chldstdy. Accessed 15.05.09

Gurwitch, R. H., Fernandez, S., Pearl, E., \& Chung, G. (2013). Utilizing parent-child interaction therapy to help improve the outcome of military families. Children, Youth, and Families Newsletter,. http://www.apa.org/pi/families/resources/newsletter/2013/01/parent-child-interaction.aspx. Accessed 06.03.14

Harwood, M. D., \& Eyberg, S. M. (2006). Child-Directed Interaction: Prediction of change in impaired mother-child functioning. Journal of Abnormal Child Psychology, 34, 335-347.

Ho, L. K. L. (2004). The treatment effectiveness of Parent-Child Interaction Therapy with depressed mother-child dyads. Unpublished Doctoral Dissertation.

Institute for Healthcare Improvement. (2003). The Breakthrough Series: IHI's collaborative model for achieving breakthrough improvement. IHI Innovation Series white paper. Boston: Author.

Kaufman Best Practices Project. (2004). Kaufman Best Practices Project Final Report: Closing the quality chasm in child abuse treatment; identifying and disseminating best practices.. http://www.chadwickcenter.org/Documents/Kaufman\%20Report/ChildHosp-NCTAbrochure.pdf. Accessed 01.02.15

Kolko, D. J., \& Swenson, C. C. (2002). Assessing and treating physically abused children and their families: A cognitive behavioral approach. Thousand Oaks, CA: Sage Publications.

Kupersmidt, J. B., Bryant, D., \& Willoughby, M. (2000). Prevalence of aggressive behaviors among preschoolers in Head Start and community child care programs. Behavioral Disorders, 26, 42-52

The National Child Traumatic Stress Network. (2015). Think Trauma.. Retrieved from http://www.nctsn.org/products/think-trauma-training-staff-juvenilejustice-residential-settings. Accessed 26.02.15

The National Child Traumatic Stress Network. (2010). Caring for children who have experienced trauma: A guide for resource parents. http://www.nctsn.org/products/caring-for-children-who-have-experienced-trauma. Accessed 26.02.15

Pearl, E. S. (2009). Parent management training for reducing oppositional and aggressive behavior in preschoolers. Aggression and Violent Behavior, 14 , 295-305.

Pearl, E., Thieken, L., Olafson, E., Boat, B., Connelly, L., Barnes, J., \& Putnam, F. (2012). Effectiveness of community dissemination of parent-child interaction therapy. Psychological Trauma: Theory, Research, Practice, and Policy, 4(2), 204-213.

Putnam, F. W. (2006). The impact of trauma on child development. Juvenile and Family Court Journal, 57(1), 1-11.

Rivard, J. C., Bloom, S. L., Abramovitz, R., Pasquale, L. E., Duncan, M., McCorkel, D., \& Gelman, A. (2003). Assessing the implementation and effects of a trauma-focused intervention for youths in residential treatment. Psychiatric Quarterly, 74, 137-154.

Rubin, D. M., O’Reilly, A. O., Luan, X., \& Localio, A. R. (2007). The impact of placement stability on behavioral well-being for children in foster care. Pediatrics, 119(2), 336-344.

Spector, L., Polka, L., Toy, S., Hoffman, A., Nyp, S., \& Andrews, S. (2012). Teaching parenting skills one resident at a time. Presented at the 12th Annual PCIT Conference. Sacramento, CA.

Tiano, J. D., \& McNeil, C. B. (2006). Training head-start teachers in behavior management using parent child interaction therapy: A preliminary investigation. Journal of Early and Intensive Behavioral Intervention, 2, 220-233.

Urquiza, A. J., \& McNeil, C. B. (1996). Parent-child interaction therapy: An intensive dyadic intervention for physically abusive families. Child Maltreatment, 1,132-141.

U.S. Department of Health and Human Services, Administration for Children and Families, Administration on Children, Youth and Families, Children's Bureau. (2013). Child maltreatment 2012.. http://www.acf.hhs.gov/programs/cb/research-data-technology/statistics-research/child-maltreatment. Accessed 02.01 .15

Webster-Stratton, C., \& Reid, M. J. (2003). Treating conduct problems and strengthening social and emotional competence in young children: The Dina Dinosaur Treatment Program. Journal of Emotional and Behavioral Disorders, 11(3), 130-143.

Webster-Stratton, C., \& Hammond, M. (1998). Conduct problems and level of social competence in Head Start children: Prevalence, pervasiveness and associated risk factors. Clinical Child Psychology and Family Psychology Review, 1, 101-124. 